\title{
Multibeam scanning electron microscope: Experimental results
}

\author{
A. Mohammadi-Gheidari, ${ }^{\text {a) }}$ C. W. Hagen, and P. Kruit \\ Delft University of Technology, Lorentzweg 1, 2628 CJ Delft, The Netherlands
}

(Received 14 July 2010; accepted 23 August 2010; published 5 November 2010)

\begin{abstract}
The authors present the first results obtained with their multibeam scanning electron microscope. For the first time, they were able to image 196 (array of $14 \times 14$ ) focused beams of a multielectron beam source on a specimen using single beam scanning electron microscope (SEM) optics. The system consists of an FEI Novanano 200 SEM optics column equipped with a multielectron beam source module. The source module consists of the multibeam source and an accelerator lens. In the multibeam source, the wide angle beam of a high brightness Schottky source is divided into 196 beamlets and focused by an aperture lens array. The accelerator lens is positioned on the image plane of the multibeam source to direct the beams toward the SEM column. The array of source images is further imaged by the SEM magnetic lenses, and the beam opening angle is defined at the variable aperture of the SEM. The system is designed to deliver $14 \times 14$ arrays of beamlets with a minimum probe size of $1 \mathrm{~nm}$. In this article, the performance of the system is examined for a fixed magnification case. (ㅇ 2010 American Vacuum Society. [DOI: 10.1116/1.3498749]
\end{abstract}

\section{INTRODUCTION}

There is a growing demand to make sub-50 $\mathrm{nm}$ features in laboratories and the semiconductor industry. To fabricate and inspect such small structures, light based systems can no longer be used. Charged particle lithography and microscopy tools provide the highest resolution, but they are not a competitive alternative because of their low throughput. The latter is a direct consequence of the limited probe current, $I$, that can be achieved in a small probe, as given by

$$
I=B_{r} \pi^{2} \alpha_{i}^{2} V d_{g}^{2} / 4,
$$

where $B_{r}$ is the reduced brightness of the electron source, $d_{g}$ is the geometrical source image size, $\alpha_{i}$ is the half opening angle of the probe, and $V$ is the acceleration voltage. Any increase in current will degrade the resolution due to the limited $B_{r}$ of the electron source. Moreover, there is a limit to the probe current for a given resolution, even for high $B_{r}$ sources, because of the statistical Coulomb interactions. Multibeam systems can enhance the throughput by several orders of magnitude. Many approaches have been tried to make multielectron beam systems over the past decades. ${ }^{1-11}$ One of the major challenges in multielectron beam systems is the electron source. To create multiple beams, different methods are available. In the single column approach, multiple beams are created either by using multiple sources ${ }^{4-6}$ or by using a single source that is split into multiple beamlets using apertures. ${ }^{7-12}$ Multiple sources are either photocathodes or cold field emitter arrays. Photocathodes have problems with poor current stability, short lifetime, and low brightness. ${ }^{12-17}$ Cold field emitters, on the other hand, are promising candidates due to their high brightness, small virtual source size, and low energy spread. They can be produced easily and cost effectively in a microfabricated array. However, years of research and investment have not yet pro-

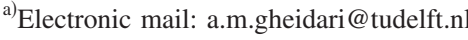

duced emitters that are sufficiently stable and reproducible to be useful in multibeam systems. ${ }^{18-21}$

As mentioned earlier, in the single source approach, the wide angle beam is split into many sub-beams. Two kinds of sources have been used so far: thermionic sources, such as $\mathrm{LaB}_{6}$, and CRT-type sources as used by Mapper (e.g., Ref. 22). Both emitters provide a very high current, but for high resolution applications, the brightness is too low.

We have developed a multielectron beam scanning electron microscope (MBSEM) as a tool for fast and high resolution electron beam induced deposition (EBID). This system is able to deliver 196 beamlets, each of which is focused down to a $1 \mathrm{~nm}$ spot, similar to state of the art single beam SEMs. Our system uses a $\mathrm{ZrO} / \mathrm{W}$ Schottky source, a source with a high brightness and good current stability. ${ }^{23,24}$ Recalling Eq. (1), this means we can achieve $32 \mathrm{pA}$ current in a 1 $\mathrm{nm}$ spot. Depending on the pattern to be written, this system enhances the writing speed up to $196 \times$. A simple example shows the importance of MBSEM as a high resolution and fast EBID system: suppose we make a $10 \times 10 \mu \mathrm{m}^{2}$ array of $1 \mathrm{~nm}^{3}$ dots at a $10 \mathrm{~nm}$ pitch using a dose of $4 \mathrm{pC} / \mathrm{nm}^{3}$, and a beam current of $25 \mathrm{pA}$. With a single beam SEM, the total writing time is almost 2 days of continuous writing. With a MBSEM, it takes only $20 \mathrm{~min}$. This system is mainly developed for high throughput fabrication of sub-10 nm structures by EBID, where resist based electron beam lithography fails. ${ }^{25}$ It can also be used for high throughput electron beam lithography and inspection (the latter, of course, only after a special detector has been developed). The aim of this article is to present the performance of the MBSEM.

\section{MBSEM SYSTEM DESIGN}

A very brief description of the electron optical working principle of the MBSEM is given here. The system consists of an FEI Novanano 200 SEM equipped with the multielectron beam source module. Figure 1(a) shows a schematic overview of the electron optical system in the MBSEM. As a 


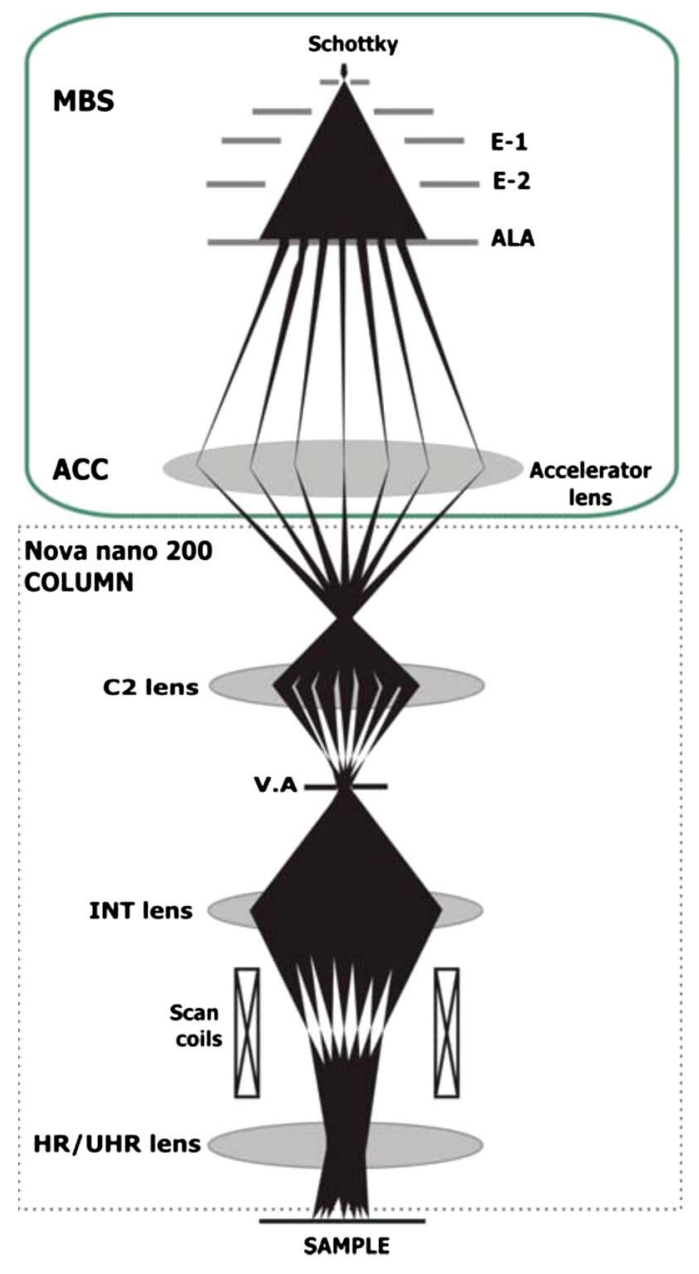

(a)

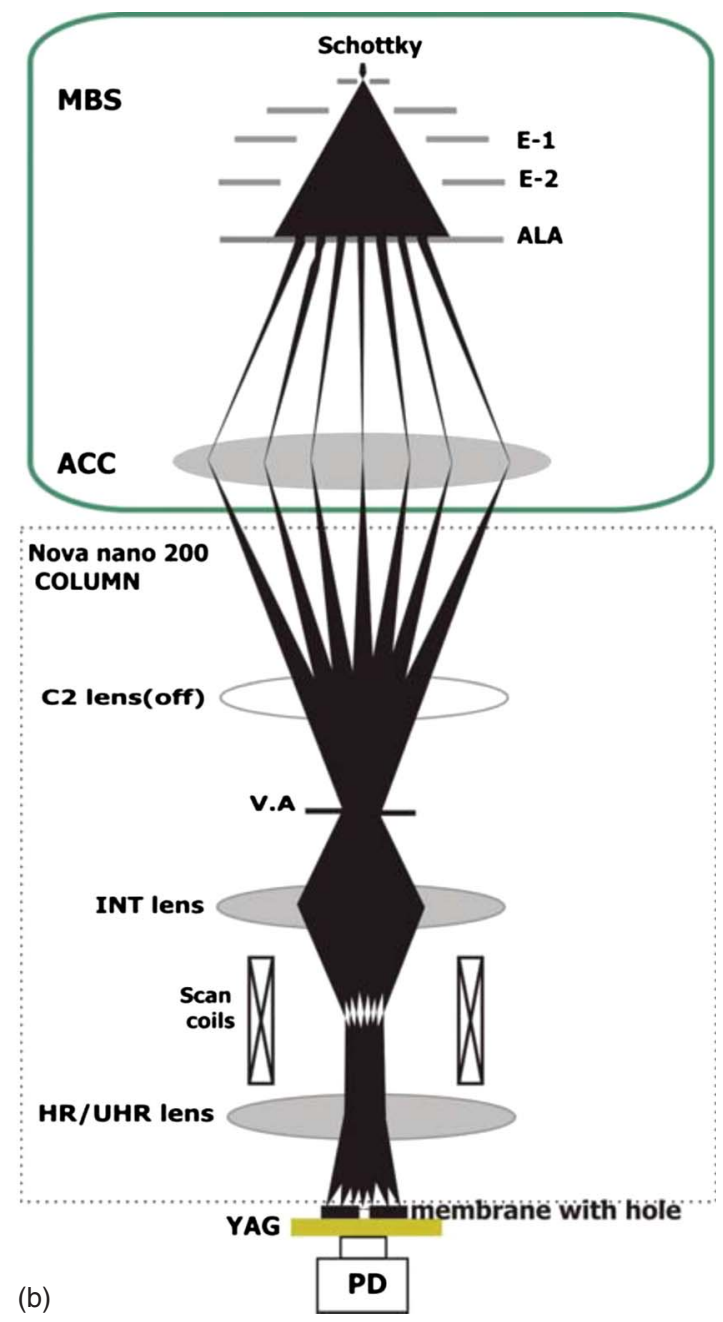

FIG. 1. (Color online) (a) Schematic overview of the electron optical system in the MBSEM. (b) Schematic illustration of the MBSEM with a fixed magnification (C2 lens off); the combined setup of "membrane with hole/YAG /PD" is used to measure the individual beam size.

consequence of using a single optical column to image all beamlets, a common crossover of all beams may degrade the system performance at high currents due to the statistical Coulomb interactions. Because of its high resolution application, the total current in the MBSEM is relatively low, and the Coulomb interaction in the crossovers is less severe. In fact, these crossovers can even be of use to change the system magnification (first crossover) or to correct the astigmatism of all beams simultaneously (second or third crossover) using a single stigmator.

The multibeam source module is composed of two crucial components: the multibeam source (MBS) and the accelerator lens (ACC). In the MBS, the emission cone of a high brightness Schottky emitter is split into an array of focused beams by an aperture lens array (ALA). The ALA is a combination of two mechanical electrodes and an array of apertures of $18 \mu \mathrm{m}$ diameter with a $25 \mu \mathrm{m}$ pitch microfabricated in a thin $\mathrm{Si}$ membrane. It is uniquely designed to correct the field curvature, to have a low spherical aberration, and to nullify the chromatic deflection error. ${ }^{26}$ The single aperture array used in the MBS avoids any precise alignment of two or more electrodes, which is the case, for instance, in a micro-Einzel lens array. ${ }^{11,27}$

In the MBS, multiple images of the source are positioned on the object principle plane of the ACC to avoid chromatic deflection aberration and to minimize the off-axial aberration of the ACC. The accelerator lens directs the beams toward the column and creates the first common crossover of all the beams in the Coulomb tube (CT). By changing the strength of the ACC and thereby moving the position of the crossover, the system magnification can be changed. ${ }^{28}$

This crossover is further imaged by the magnetic condenser lens C2 onto the variable aperture (VA) that acts as a current limiting aperture. With the VA, it is possible to manipulate the opening angle and, consequently, the probe current. The intermediate magnetic lens is used to image the VA onto the coma free plane of the objective lens to suppress the off axis aberrations of the high resolution (HR)/ultrahigh resolution (UHR) objective lens. Further demagnification of the probes will be done by the UHR/HR lens. 
TABLE I. Calculated electron optical parameters of the system with a fixed magnification and a $1 \mathrm{~nm}$ resolution in UHR mode. (Note that $C_{s t}$ and $C_{c t}$ stand for total spherical and chromatic aberration coefficients, respectively.)

\begin{tabular}{lc}
\hline \hline Parameter & Value (UHR) \\
\hline Total magnification (Mtot) & 0.0162 \\
Pitch at the wafer (nm) & 360 \\
Geometrical spot (nm) & 0.49 \\
$C_{s t}$ image side (mm) & 5.43 \\
$C_{c t}$ image side (mm) & 3.07 \\
Working distance (mm) & 5 \\
Acceleration voltage $(\mathrm{keV})$ & 15 \\
\hline \hline
\end{tabular}

The MBS is designed to create an array of focused beams in the ACC plane, with a geometrical spot size of $95 \mathrm{~nm}$ at a pitch of $70 \mu \mathrm{m}$. The pitch to geometrical probe size ratio is conserved throughout the system. For a landing energy of $1500 \mathrm{eV}$ in the ACC plane, the total current delivered by the MBS is $157 \mathrm{nA}$, or $0.8 \mathrm{nA}$ per beamlet [with a typical brightness of $5 \times 10^{7} \mathrm{~A} /\left(\mathrm{m}^{2} \mathrm{sr} \mathrm{V}\right)$ and a FW50 energy spread of $0.5 \mathrm{eV}$ for the Schottky source $\left.{ }^{27}\right]$. This current can be simply measured in the CT. Depending on the required resolution, the current is further cut off by the VA.

For applications with a fixed total magnification, the $\mathrm{C} 2$ lens can be switched off. The imaging sequence of this particular feature of the MBSEM is illustrated in Fig. 1(b), which is the situation of interest in this article. This configuration is designed to give a $1 \mathrm{~nm}$ spot at the wafer in UHR mode. The detailed electron optical calculation will be published elsewhere. ${ }^{28}$ Table I summarizes the calculated electron optical parameters of the system with a fixed magnification and a $1 \mathrm{~nm}$ resolution in the UHR mode. Figure 2 shows the calculation results of the total axial spot size, and different aberration contributions in it as a function of the VA size. As can be seen from the figure, the smallest axial probe size of dtot $=1.17 \mathrm{~nm}$ is obtained for a VA of $20 \mu \mathrm{m}$. The corresponding optimum opening angle is then $\alpha_{i}=8.5 \mathrm{mrad}$.

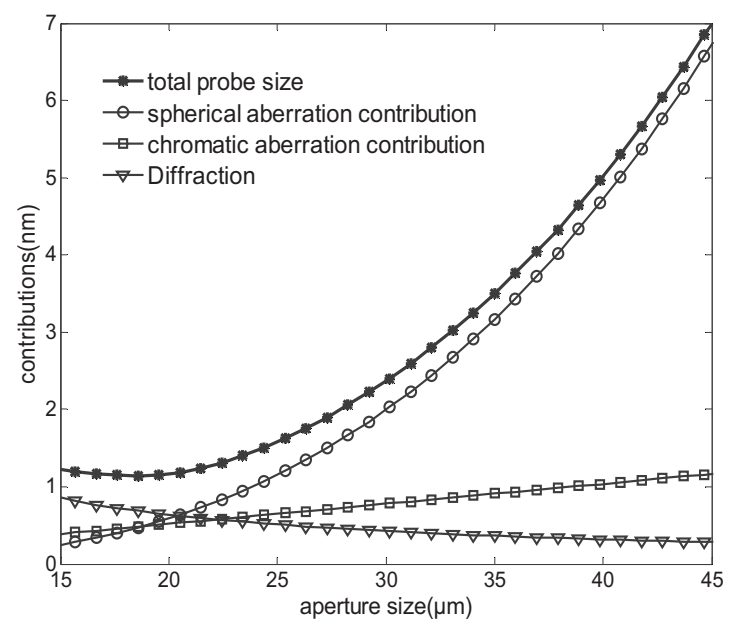

FIG. 2. Calculation results of the total axial spot size and its different axial aberration contributions as a function of the VA size.

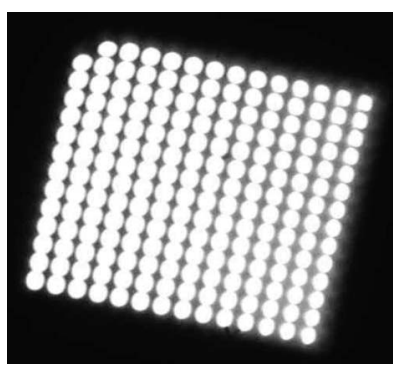

(a)

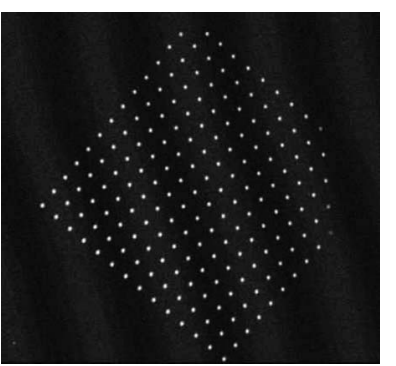

(b)
FIG. 3. (a) Direct image of 196 beams in the chamber onto a YAG screen and recorded with the CCD camera (scale of $1 \times 1 \mathrm{~mm}^{2}$ ). (b) MBSEM image of single aperture (pitch $\approx 0.4 \mu \mathrm{m}$ ).

\section{MBSEM PERFORMANCE}

The very first challenge is to image all beamlets onto the specimen. To visualize the beams at the specimen level, we mounted an yttrium aluminum garnet (YAG) screen and a charged coupled device (CCD) camera at the specimen position. Figure 3(a) shows an image of the defocused array of beams in the chamber. This is the very first demonstration that we can image $196(14 \times 14)$ beamlets using a single electron optical column.

Now, we need to characterize the beamlets in terms of current, size, pitch, and eventually brightness (not a subject of this article). Unlike a single beam system, measuring the individual beamlet size of the MBSEM is not straightforward because there is no full control over each beamlet. In a single beam system, one can scan the beam across a sharp edge (Knife edge method), and the beam size is then determined by measuring the width of the intensity profile between two fixed levels (normally 25\%-75\% or a different portion depending upon the definition). Alternatively, the resolution of the system can be quantified using the edge sharpness of features in the secondary electron images, e.g., nanogold balls (image processing). In our MBSEM, all beamlets can only be scanned simultaneously. This means that the signal of one beamlet cannot be distinguished from the others. To overcome this problem, all beams are scanned simultaneously over an aperture. This aperture is a hole with a diameter of $220 \mathrm{~nm}$ made by focused ion beam (FIB) drilling in a silicon nitride $\left(\mathrm{Si}_{3} \mathrm{~N}_{4}\right)$ membrane covered with titanium (Ti) on both sides. The diameter of the hole is smaller than the pitch between the beamlets, and the membrane is thick enough $(\approx 800 \mathrm{~nm})$ to stop electrons of up to $20 \mathrm{keV}$. By integrating a YAG screen and a photodiode under the aperture, and scanning the beamlets over the aperture, each beam builds up a scanning transmission electron microscope-like image of the aperture. A schematic of this experimental setup is illustrated in Fig. 1(b). Analyzing the intensity profile of the transmitted current for each beamlet gives a first measure of the size and pitch between the beams. Figure 3(b) shows the MBSEM image of the single aperture. Figure 4 shows a picture with an example of the image analysis, in which the measured pitch is about $400 \mathrm{~nm}$ and the beam size is $50 \mathrm{~nm}$. The measured value for the pitch is close to its predicted value of $360 \mathrm{~nm}$ (see Table I), but the beam size is far from 


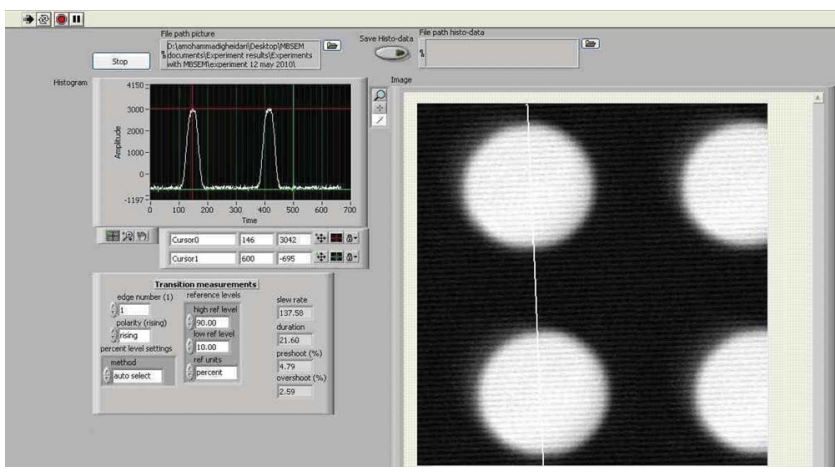

FIG. 4. (Color online) Picture of the program used to analyze the beam size and pitch of the MBSEM.

its predicted value of $1 \mathrm{~nm}$ for a VA of $20 \mu \mathrm{m}$. This is not surprising. As in the knife edge measurement method, the quality of the aperture plays an important role. In fact, the accuracy of the beam size measurement mainly depends on the quality of the edge over which the beam is scanned, and the high aspect ratio aperture used here is far from ideal.

To improve the measurement, a rod of $20 \mathrm{~nm}$ width is grown across the hole using EBID from a methylcyclopentadienyl-platinum-tri-methyl $(\mathrm{MeCpPtMe})$ precursor. This already provides a much sharper edge. Figures 5(a) and 5(b) show some typical results of this experiment where the $20 \mathrm{~nm}$ rod is clearly imaged by all beams. The beam size is estimated by measuring the width of the intensity profile between two fixed levels (25\%-75\%) in the line profile. As before, the measured pitch between the beams is $400 \mathrm{~nm}$, but the measured beam sizes are around $14 \mathrm{~nm}$. We have seen that after scanning the beams over this sample, the EBID rod became thinner and shorter. It was disconnected from one side of the hole [see Fig. 6(a)]. Figure 6(b) shows a result of the beam size analysis with a zoomed in image of a rod (inset) of width between 15 and $18 \mathrm{~nm}$. In this case, the average measured beam size $(25 \%-75 \%$ value $)$ is $10 \mathrm{~nm}$. The measured beam size is clearly an upper limit because of the somewhat poor image detection method with the photodiode.

The total current delivered by the MBS is measured at the $\mathrm{CT}$ of the system. Prior to the measurement, all beams were deflected using beam shift/tilt coils to the side of the CT to
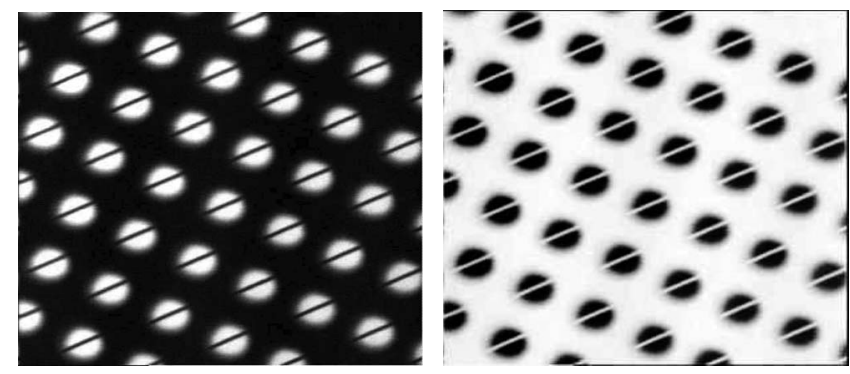

(a)

(b)

FIG. 5. (a) Typical MBSEM transmission image of a single $220 \mathrm{~nm}$ diameter aperture with a $20 \mathrm{~nm}$ width EBID rod. (UHR mode). (b) The same image with inversed contrast.

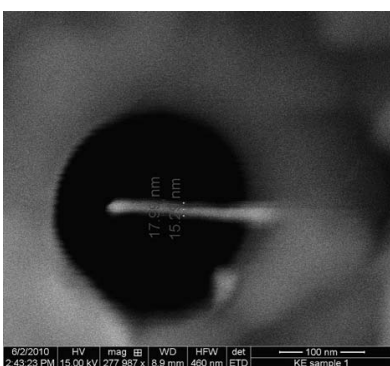

(a)

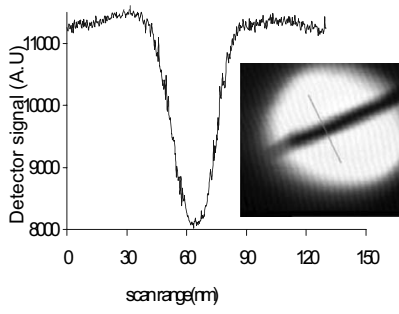

(b)
FIG. 6. (a) SEM image of a FIB drilled aperture with a broken EBID rod attached. The width of the rod is $15-18 \mathrm{~nm}$. (b) Intensity profile across an EBID-rod and (inset) a zoomed in transmission MBSEM image of the rod.

make them all contribute to the measurement. To measure the total current delivered at the specimen level, all beams are focused into a Faraday cup, and the total current of the system is measured for different VAs of 20,30, 40, and $50 \mu \mathrm{m}$. Figure 7(a) shows the calculated total current as a function of VA and the measured values. It is observed that they are very close to the predicted values. From Fig. 7(b), it can be seen that the current distribution is almost uniform. The graph shows an integrated line profile of the indicated row (inset) in the array of beams. The area under each peak in this figure shows the total current confined in each probe. The maximum fluctuation between the areas was only 5\% within a row. Similar results were obtained for different rows. This shows that the total current is uniformly distributed over 196 beams. For VA=20 $\mu \mathrm{m}$, for example, the measured total current is $6.1 \mathrm{nA}$; i.e., the current per beamlet is $31.1 \mathrm{pA}$, and the calculated current per beamlet is $31.4 \mathrm{pA}$. The total current delivered by the MBS to the system can be measured in the CT and is found to be $150 \mathrm{nA}$, which is also very close to the predicted value.

The current stability is also a very important factor. The total current fluctuation was captured for more than $2 \mathrm{~h}$ of operation, and it turned out to be negligible. Also, the long term stability of the multibeam source module is very good, as it is already reliably operating now for more than 1 year.

To further improve the beam size measurements, we intend to install a scanning transmission electron microscopy (STEM) detector and use angular dark field imaging.

\section{SINGLE BEAM PERFORMANCE OF THE MBSEM}

As mentioned before, it is possible to move the first common crossover of the beamlets by changing the ACC strength. As schematically illustrated in Fig. 8(a), it is possible to position the crossover such that all beams, but one, are stopped in the pumping apertures located in the $\mathrm{C} 2$ lens. Using beam shift/tilt coils, this beam can be further aligned and directed toward the SEM column.

Figure 8(b) shows a typical result of MBSEM performance as a standard single beam SEM. This image is made with a randomly chosen off axis beamlet by beam shift/tilt coils. It should be noted that the same experiment has been carried out with some other beamlets and that they revealed 

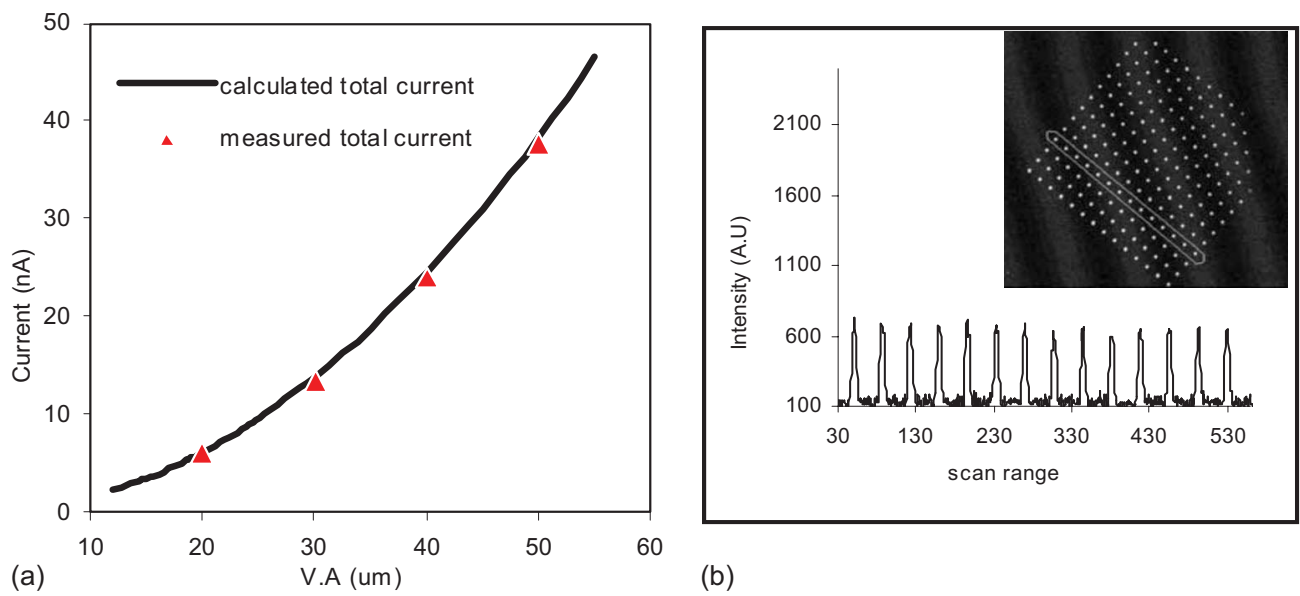

(b)

FIG. 7. (Color online) (a) Calculated total current of 196 beams and the measured values as a function of the VA. (b) The graph shows an integrated intensity profile of the row of beamlets as indicated in the inset, which shows the array of beamlets.

the same imaging properties. The current measured for the beam was $43 \mathrm{pA}$, and the resolution is measured to be less than $2 \mathrm{~nm}$ using a proprietary image processing program from FEI that essentially measures edge resolution of sharp features in the image. The resolution can also be estimated from the gap between tin balls in the image, in which a spacing less than $2 \mathrm{~nm}$ between balls is still distinguishable.

\section{CONCLUSION}

We have developed a MBSEM dedicated for high resolution, high throughput lithography, and microscopy. For the first time, we were able to image 196 beams of a multielectron beam source on a specimen using single beam SEM optics. The source has a stable operation now for more than a year. The probe current and the probe size measurement results show a promising performance for the MBSEM. The measured pitch, the current per beam, and the total current in the $\mathrm{CT}$ are in good agreement with the predicted values. The current per beam is comparable with that of a single beam
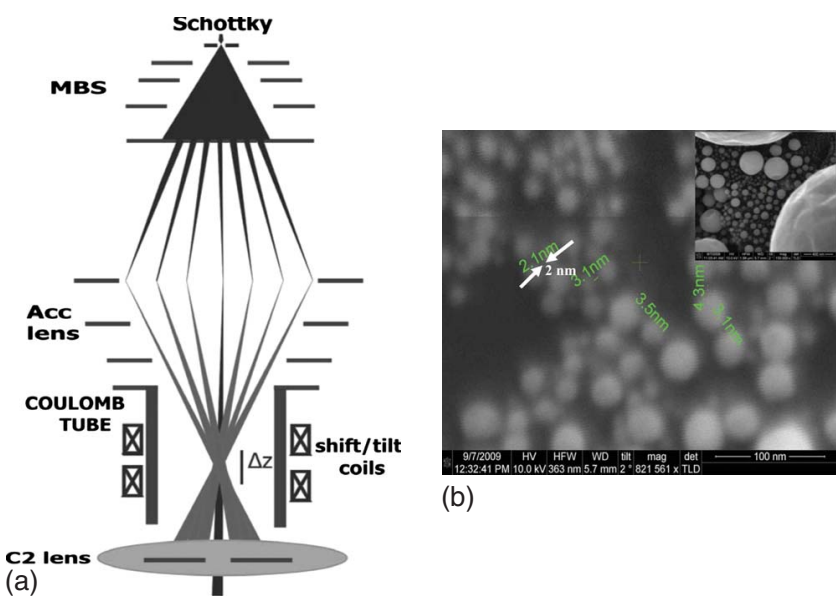

(b)

FIG. 8. (Color online) (a) Working principle of single beam operation of the MBSEM [only the indicated beam (black) is not blanked]. (b) SEM image of tin balls made by a randomly chosen off axis beam of the MBSEM. (Inset) a slightly zoomed out image (scale bar is $400 \mathrm{~nm}$ ).
SEM. We are confident that the beam sizes are also quite close to the calculated values, as can be inferred from the single beam performance of the system. The main limitation to measure the beam size of all beamlets was the use of the photodiode as a detector. We intend to improve on this using a STEM detector in the future.

\section{ACKNOWLEDGMENTS}

The author A.M.-G. would like to thank the FEI Company for providing valuable information. He would also like to thank J. Nonhebel, F. Berwald, J. de Looff, C. Th. H. Heerkens, V. G. Kutchoukov, and C. Post, technicians from Charged Particle Optics group and A. C. Wattjes, C. Barends, and G. Schotte from Demo for their support.

${ }^{1}$ B. Roelofs and J. Barth, Microelectron. Eng. 2, 259 (1984).

${ }^{2}$ M. McCord, J. Vac. Sci. Technol. B 15, 2125 (1997).

${ }^{3}$ T. Chang, M. Mankos, K. Lee, and L. Muray, Microelectron. Eng. 57-58, 117 (2001).

${ }^{4}$ E. Yin, A. D. Brodie, F. C. Tsai, G. X. Guo, and N. W. Parker, J. Vac. Sci. Technol. B 18, 3126 (2000).

${ }^{5}$ S. Tanimoto, Y. Someda, M. Okumura, H. Ohta, Y. Sohda, and N. Saitou, Jpn. J. Appl. Phys., Part 1 42, 6672 (2003).

${ }^{6}$ S. T. Coyle et al., J. Vac. Sci. Technol. B 22, 501 (2004).

${ }^{7}$ H. Yasuda, S. Arai, J.-I. Kai, Y. Ooae, T. Abe, S. Maruyama, and T. Kiuchi, J. Vac. Sci. Technol. B 14, 3813 (1996).

${ }^{8}$ G. Winograd, V. Krishnamurthi, R. Garcia, L. H. Veneklasen, M. Mankos, and F. Pease, J. Vac. Sci. Technol. B 18, 3052 (2000).

${ }^{9}$ M. Muraki and S. Gotoh, J. Vac. Sci. Technol. B 18, 3061 (2000).

${ }^{10}$ N. Nakasuji, S. Yoshikawa, T. Satake, and N. Noji, Jpn. J. Appl. Phys., Part 1 44, 5570 (2005)

${ }^{11}$ O. Kamimura et al., J. Vac. Sci. Technol. B 25, 140 (2007).

${ }^{12}$ G. Schwind, G. Magera, and L. Swanson, J. Vac. Sci. Technol. B 24, 2897 (2006).

${ }^{13}$ M. Mankos, S. Coyle, A. Fernandez, A. Sagle, P. Allen, W. Owens, J. Sullivan, and T. Chang, J. Vac. Sci. Technol. B 18, 3010 (2000).

${ }^{14}$ X. Jiang, C. Berglund, A. Bell, and W. Mackie, J. Vac. Sci. Technol. B 16, 3374 (1998).

${ }^{15}$ M. Wieland, B. Kampherbeek, P. Addessi, and P. Kruit, Microelectron. Eng. 57-58, 155 (2001).

${ }^{16}$ D. Schroder, R. Thomas, J. Vine, and H. Nathanson, IEEE Trans. Electron Devices 21, 785 (1974).

${ }^{17}$ T. Teepen, A. van Veen, H. van't Spijker, S. Steenbrink, A. van Zuuk, C. Heerkens, M. Wieland, N. van Druten, and P. Kruit, J. Vac. Sci. Technol. B 23, 359 (2005). 
${ }^{18}$ W. Hofmann, L.-Y. Chen, and N. MacDonald, J. Vac. Sci. Technol. B 13, 2701 (1995).

${ }^{19}$ Y. Yamaoka, S. Kanemaru, and J. Itoh, Jpn. J. Appl. Phys., Part 1 35, 6626 (1996).

${ }^{20}$ J.-Y. Park, J. Lera, M. Yakshin, S. Choi, Y. Lee, K. Chun, J. Lee, D. Jeon, and Y. Kuk, J. Vac. Sci. Technol. B 15, 2749 (1997).

${ }^{21}$ G. Guo, K. Tokunaga, E. Yin, F. Tsai, A. Brodie, and N. Parker, J. Vac. Sci. Technol. B 19, 862 (2001).

${ }^{22}$ A. J. van den Brom, A. H. V. van Veen, W. M. Weeda, G. Z. M. Berglund, M. Wieland, and P. Kruit, J. Vac. Sci. Technol. B 25, 2245 (2007).
${ }^{23}$ M. S. Bronsgeest, Ph.D. thesis, Delft University of Technology, 2009.

${ }^{24}$ M. J. Fransen, M. H. F. Overwijk, and P. Kruit, Appl. Surf. Sci. 146, 357 (1999).

${ }^{25}$ A. E. Grigorescu M. C. van der Krogt E. W. J. M van der Drift, and C. W. Hagen, J. Micro/Nanolith. MEMS MOEMS 7, 013005 (2008).

${ }^{26}$ Yanxia Zhang and Pieter Kruit, Phys. Procedia 1, 553 (2008).

${ }^{27}$ M. J. van Bruggen, Ph.D. thesis, Delft University of Technology, 2008.

${ }^{28}$ A. Mohammadi Gheidari and P. Kruit, Proceedings of the CPO-8 Conference, Singapore, 12-16 July, 2010 (unpublished). 